\title{
A Computationally Efficient Method for Full-Core Conjugate Heat Transfer Modeling of Sodium Fast Reactors
}

\author{
Rui Hu and Yiqi Yu \\ Nuclear Engineering Division, Argonne National Laboratory, \\ 9700 S. Cass Avenue, Argonne IL 60439, USA \\ Tel: +1-630-252-1461, Email: rhu@anl.gov
}

\begin{abstract}
For efficient and accurate temperature predictions of sodium fast reactor structures, a 3-D full-core conjugate heat transfer modeling capability is developed for an advanced system analysis tool, SAM. The hexagon lattice core is modeled with 1-D parallel channels representing the subassembly flow, and 2-D duct walls and inter-assembly gaps. The six sides of the hexagon duct wall and near-wall coolant region are modeled separately to account for different temperatures and heat transfer between coolant flow and each side of the duct wall. The Jacobian Free Newton Krylov (JFNK) solution method is applied to solve the fluid and solid field simultaneously in a fully coupled fashion. The 3-D full-core conjugate heat transfer modeling capability in SAM has been demonstrated by a verification test problem with 7 fuel assemblies in a hexagon lattice layout. Additionally, the SAM simulation results are compared with RANS-based CFD simulations. Very good agreements have been achieved between the results of the two approaches.
\end{abstract}

Keywords: conjugate heat transfer, system thermal-hydraulics, JFNK, sodium fast reactor

\section{INTRODUCTION}

Detailed core-wide temperature predictions of sodium-cooled fast reactors (SFR) need to be performed in order to ensure that all the design criteria are satisfied, such as fuel, cladding, and sodium outlet temperatures under various conditions. Additionally, one important design requirement for SFR is the knowledge of the temperature on the hexagonal ducts for a thermo-mechanical analysis. This is particular important to ensure the passive safety of the reactor under the unprotected accident conditions if the reactor control system fails to function and the reactivity feedback from structural deformation such as core radial expansion is significant (Chang et al., 2005). This information requires a good evaluation of the inter-assembly flow and heat transfer in this region. The physical phenomena are particularly complicated and require a reliable modeling of the whole core including the inter-assembly region. For repeated detailed core-wide temperature calculations, a computationally efficient modeling approach is necessary.

Multiple levels of modeling are possible for the analysis of the whole core thermal hydraulic behavior, depending on the purpose of the calculation and the accuracy required. Generally, the 3D full core modeling using the computational fluid dynamics (CFD) approach is prohibitively expensive. In order to reduce the amount of computational effort but to maintain a reasonable accuracy, a low resolution RANS approach is developed by $\mathrm{Hu}$ and Fanning (2011). In this approach a momentum source model was developed to mimic the effects of the wire-wraps without explicitly modeling the geometrical representation of the wire. Similar low grid resolution approaches of rod bundle simulation have been 
reported by Roelofs et al. (2012) and Viellieber and Class (2015). Such low-resolution approaches are required to simulate the overall behavior of the coolant flow in a complete fuel assembly or even multiple fuel assemblies interacting with each other. The low-resolution approaches can provide more local details than typically can be deducted from subchannel simulations, while maintaining a reasonable accuracy compared to detailed CFD analyses for a complete fuel assembly that are too costly for engineering purposes. The low-resolution approach can be very useful to study the effects of partial flow blockage and local deformations, but still too expensive for repeated full-core simulations.

Over the past several decades, much effort has been made to develop the sub-channel analysis codes to predict the detailed coolant temperatures for all the sub-channels in a fuel assembly or reactor core. More recent efforts include the development of SE2-ANL (Yang and Yacout, 1995), MATRA-LMR (Kim et al., 2002) and TRIO_U subchannel module (Tenchine et al., 2012). Yu et al. (2015) developed a porous medium modeling approach to predict SFR duct wall temperatures, which is much less computationally expensive than conventional CFD simulations that explicitly represent the wire-wrap and fuel pin geometry. However, even the porous medium or subchannel description of each subassembly and the inter-assembly space is still too computational expensive for quotidian analyses.

System analysis is favorable to describe the global behavior of the whole core during transient situations. In traditional reactor safety analysis, the reactor core is modeled with several 1D parallel channels to take into account the main different core regions, with a possible by-pass channel to simulate amassed flow rate in the inter-assembly zone (Tenchine, 2010). However, it is very cumbersome to obtain the detailed temperature predictions of inter-assembly gaps and duct walls, which requires a full-core one-to-one representation of fuel assemblies and inter-assembly gaps.

For efficient and accurate full-core heat transfer calculations in SFR, especially for structure temperature predictions, a 3-D full-core conjugate heat transfer modeling capability has been developed for an advanced system analysis tool, SAM, being developed at Argonne National Laboratory. The hexagon lattice core can be modeled with 1-D parallel channels representing the subassembly flow and 2-D duct walls and intra-assembly gaps. Note that each fuel assembly can be modeled as multiple channels and the 6 sides of the hexagon duct wall are modeled separately to account for different temperatures and heat transfer between inner assembly flow and each side of the duct wall. A core lattice model has been developed in SAM so that it can, based on very simple input descriptions, generate all the core channels and inter-assembly gaps, and build the connections among them.

Hu et al. (2015) provided an overview and update of the SAM code development. The in-depth discussion of the underlying physics models and numerical methods were report by $\mathrm{Hu}$ (2015). This paper presents a computationally efficient conjugate heat transfer modeling method in SAM for radial heat transfer in the reactor core. The 3-D full-core conjugate heat transfer modeling capability is demonstrated by a verification test problem with 7 fuel assemblies in a hexagon lattice layout. The simulation results are compared with the RANS-based CFD simulation using the commercial CFD code STAR-CCM+ (CDadapco 2014). Good agreements are achieved between the results of the two codes.

\section{SAM CONJUGATE HEAT TRANSFER MODELING}

\subsection{SAM Overview}

SAM is an advanced system analysis tool being developed at Argonne National Laboratory under the U.S. DOE's Nuclear Energy Advanced Modeling and Simulation (NEAMS) program. The code is aimed to solve the tightly-coupled physical phenomena including fission reaction, heat transfer, fluid dynamics, and thermal-mechanical response in the SFR structures, systems and components in a fully-coupled fashion but with reduced-order modeling approaches to facilitate rapid turn-around for design and safety 
optimization studies. As a new code development, the initial effort focused on developing modeling and simulation capabilities of the heat transfer and single-phase fluid dynamics responses in the SFR systems.

SAM utilizes a modern application framework MOOSE (Gaston et al., 2009), the underlying meshing and finite-element library libMesh (Kirk et al., 2006), and linear and non-linear solvers PETSc (Balay et al., 2016) to leverage the available advanced software environments and numerical methods. The physics modeling and mesh generation of individual reactor components are encapsulated as component classes in SAM along with some component specific models. A set of components has been developed based on the finite element models (for fluid flow and heat transfer), and the system simulation capabilities of general thermal-hydraulics systems have been demonstrated and benchmarked with the EBR-II tests (Hu and Sumner, 2016).

Fluid dynamics is the main physical model of the SAM code. SAM employs a one-dimensional transient model for single-phase incompressible but thermally expandable flow. The transport equations for onedimensional, single-phase flow can be described by a set of partial differential equations. The mass, momentum, and energy conservation equations are closed by the equation of state for the fluid. After some simplifications, the conservative form of the governing equations can be written in Eq. (1), and it is solved in SAM using the primitive variable based approach. The details of the finite-element model for single-phase incompressible but thermally expandable flow and the associated stabilization scheme in SAM can be found in $\mathrm{Hu}(2015)$.

$$
\begin{aligned}
& \frac{\partial \rho}{\partial t}+\frac{\partial(\rho u)}{\partial z}=0 \\
& \frac{\partial(\rho u)}{\partial t}+\frac{\partial(\rho u u+p)}{\partial z}=-\rho g-\frac{f}{D_{e}} \frac{\rho u|u|}{2} \\
& \frac{\partial(\rho H)}{\partial t}+\frac{\partial(\rho u H)}{\partial z}=q^{\prime \prime \prime} \\
& \rho=\rho(p, T)
\end{aligned}
$$

In which $f$ : the friction coefficient; $D_{e}$ : equivalent hydraulic diameter. When considering the convection heat flux from solid surface $q_{s}^{\prime \prime}, q^{\prime \prime \prime}=q_{s}^{\prime \prime} P_{h} / A_{c}$, where $P_{h}$ and $A_{c}$ respectively denote heated perimeter and cross-sectional area of the coolant channel.

Heat structures model the heat conduction inside the solids and permit the modeling of heat transfer at the interfaces between solid and fluid components. Heat structures are represented by one-dimensional or two-dimensional heat conduction in Cartesian or cylindrical coordinates. Temperature-dependent thermal conductivities and volumetric heat capacities can be provided in tabular or functional form either from built-in or user-supplied data. The modeling capabilities of heat structures can be used to predict the temperature distributions in solid components such as fuel pins or plates, heat exchanger tubes, and pipe and vessel walls, as well as to calculate the heat flux conditions for fluid components.

The thermal conduction inside the solid structures is governed by a diffusion equation:

$$
\rho C_{p} \frac{\partial T}{\partial t}-\nabla(k \nabla T)-Q^{\prime \prime \prime}=0
$$

Where $k$ is the solid thermal conductivity, and $Q^{\prime \prime \prime}$ is the volumetric internal heat source in the solid. It can be discretized in both Cartesian and cylindrical coordinates. The types of boundary conditions (BC) include: (1) Dirichlet BC, $T=T_{0}$; (2) Neumann BC, $k \nabla T=q_{0}$ "; or (3) convective $\mathrm{BC}:-k \nabla T=h$. $\left(T-T_{\text {fluid }}\right)$. 
Since SAM is built on the MOOSE framework, it relies on the Jacobian Free Newton Krylov (JFNK) solution method (Knoll and Keyes, 2004) to solve all the unknowns in a fully coupled fashion. This fully coupled solution scheme avoids the operator-splitting errors and is especially valuable for conjugate heat transfer problems in which the heat conduction in the solid is tightly coupled with the fluid flow. On the contrary, the operator-splitting methods decompose the systems of PDEs into simpler sub-problems and solve them individually in sequence and using specialized numerical algorithms, which would result in more numerical errors. Careful control of the solution schemes (iterative or prediction-correction) in the operator-splitting methods is critical for the stability, robustness, and performance of the solver. The JFNK method is a multi-level approach, using the outer Newton's iterations (nonlinear solver) and inner Krylov subspace methods (linear solver), to solve large nonlinear systems. The JFNK method has become an increasingly popular option for solving large nonlinear equation systems and multi-physics problems, as observed in a number of different disciplines (Knoll and Keyes, 2004).

In JFNK methods, the concept of 'Jacobian-free' is proposed, because deriving and assembling such large Jacobian matrices could be difficult and expensive. However, in most applications, the Krylov subspace methods require preconditioning to be efficient. This is especially true for the fluid flow and conjugate heat transfer problems. In the solution methods currently used in SAM, an approximate analytical Jacobian matrix is computed and passed to the underlying numerical solver library (PETSc) for preconditioning purposes.

\subsection{Convective Heat Transfer at Fluid-Solid Interface}

In many engineering applications, the fluid flow and solid heat conduction is coupled through convective heat transfer at the solid surfaces. The conjugated heat transfer modeling in SAM at the fluid-structure interface is shown schematically in Figure 1. The fluid is modeled as one-dimensional flow, and the solid structure is modeled as one-D or two-D heat conduction, and they exchange energy at the fluid-structure interface.

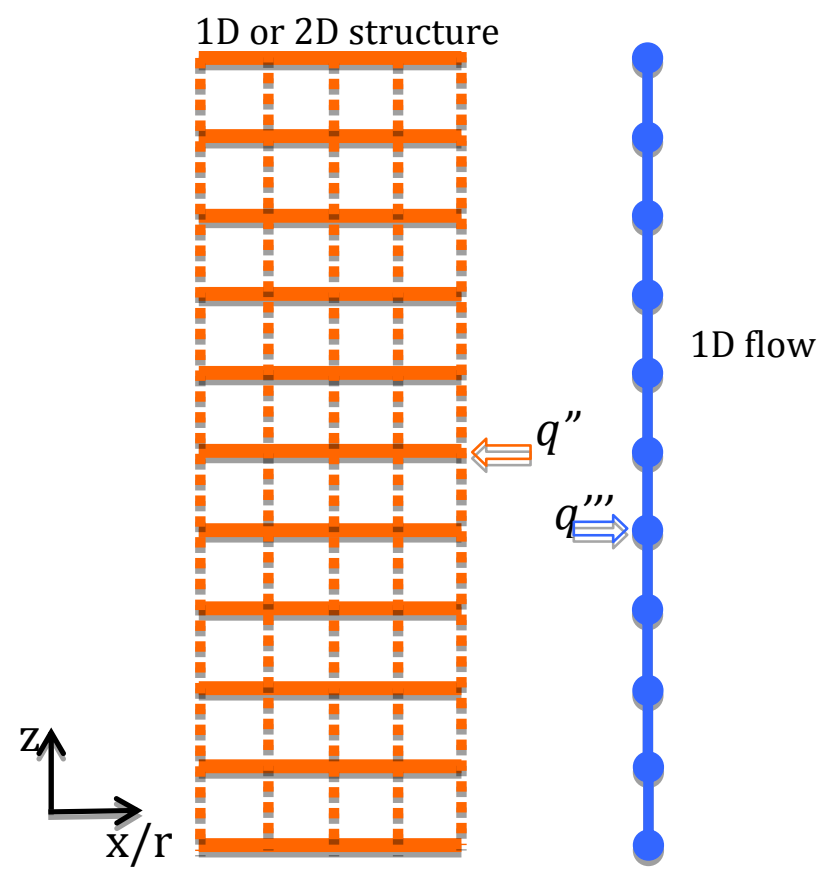

Figure 1: The Schematic of conjugate heat transfer modeling in SAM 
At the fluid-structure interface, the convective heat flux is:

$$
q^{\prime \prime}=k \nabla T=h\left(T_{w}-T_{f}\right) \text {. }
$$

In which $h$ is the heat transfer coefficient, $T_{w}$ is the wall temperature, and $T_{f}$ is the fluid temperature.

The weak form of the solid conduction equation (Eq.-2) can be written as,

$$
\left(\rho C_{p} \frac{\partial T}{\partial \mathrm{t}}, \psi\right)+(k \nabla T, \nabla \psi)-\langle k \nabla T, \psi\rangle-\left(Q^{\prime \prime \prime}, \psi\right)=0
$$

In which $(f, \psi)$ represents the volume integral, $(f, \psi)=\int_{\Omega} \psi * f d \Omega$; and $\langle f, \psi\rangle$ represents the surface integral, $\langle f, \psi\rangle=\int_{\Gamma} \psi * f d \Gamma$. For one-D radial solid element at the interface, the convective heat flux can be directly applied as boundary conditions for the one-D heat conduction equation, as required by the $\langle k \nabla T, \psi\rangle$ term in Eq. (4).

However, for fluid element, it has to be modeled as an additional heat source term in the energy conservation equation,

$$
q^{\prime \prime \prime}=h\left(T_{w}-T_{f}\right) \frac{P_{\text {heated }}}{A_{c}} .
$$

This is implemented as an additional term in the residual calculation at each fluid node (quadrature point):

$$
\Delta r_{\text {energy }}=J \mathrm{xW} \cdot q^{\prime \prime \prime} \cdot \phi=J \mathrm{x} W \cdot h\left(T_{w}-T_{f}\right) \frac{P_{\text {heated }}}{A_{c}} \cdot \phi,
$$

In which, $J x W$ is Jacobian times weight, and $\phi$ is the shape function. Eq. (6) is derived from the discretized form of $\int_{\Omega} \psi * q^{\prime \prime \prime} d \Omega$. Note the above implementation may introduce another term in the stabilized fluid model formulation. For the SUPG (Streamline Upwind Petrov-Galerkin) scheme used in SAM,

$$
\Delta r_{\text {energy }}=J \mathrm{xW} \cdot h\left(T_{w}-T_{f}\right) \frac{P_{\text {heated }}}{A_{c}} \cdot\left(\phi+\tau_{\text {supg }} \cdot \nabla \phi\right) .
$$

\subsection{Preconditioning of Convective Heat Transfer Modeling}

As mentioned above, a Krylov-type of method generally requires preconditioning to be efficient and effective. It is also well known that the closer the preconditioning matrix is to the exact Jacobian matrix, the better the convergence behavior. In SAM, an approximation of the exact Jacobian is provided to the solver as a preconditioning matrix, as the exact Jacobian matrix is very difficult to obtain and not necessary. For one-dimensional flow and heat conduction problems, tri-diagonal terms, due to spatial discretization, are included in the preconditioning matrix. Since the conjugate heat transfer is a tightly coupled phenomenon between the solid conduction and fluid flow, its Jacobian terms must be included.

The Jacobian terms represent the effect of one variable perturbation on the residuals of another variable. For convective heat transfer between fluid and structure, the Jacobian terms can be derived as:

$$
\begin{aligned}
& J\left(T_{s}, T_{s}\right)=\frac{\partial\left[h\left(T_{w}-T_{f}\right)\right]}{\partial T_{f}} \approx h \\
& J\left(T_{s}, T_{f}\right)=\frac{\partial\left[h\left(T_{w}-T_{f}\right)\right]}{\partial T_{f}} \approx-h \\
& J\left(T_{f}, T_{s}\right)=\frac{\partial\left[h\left(T_{w}-T_{f}\right) \frac{P_{\text {heated }}}{A_{C}}\right]}{\partial T_{w}} \approx-h \frac{P_{\text {heated }}}{A_{c}}
\end{aligned}
$$




$$
J\left(T_{f}, T_{f}\right)=\frac{\partial\left[h\left(T_{w}-T_{f}\right) \frac{P_{\text {heated }}}{A_{c}}\right]}{\partial T_{w}} \approx h \frac{P_{\text {heated }}}{A_{c}}
$$

In which $T_{f}$ and $T_{S}$ represent fluid and solid temperature, respectively. Note in Eq. (8) that the dependences of heat transfer coefficient, $h$, on the fluid and solid variables are neglected.

For a conjugate heat transfer problem with only one fluid block and one solid block, the shape of the preconditioning matrix looks like Figure 2, in which the circles and red dots represent the non-zero entries from the convective heat transfer between fluid and structures. A similar study on the preconditioning of JFNK method for conjugate heat transfer problem is reported in Zou et al. (2013).

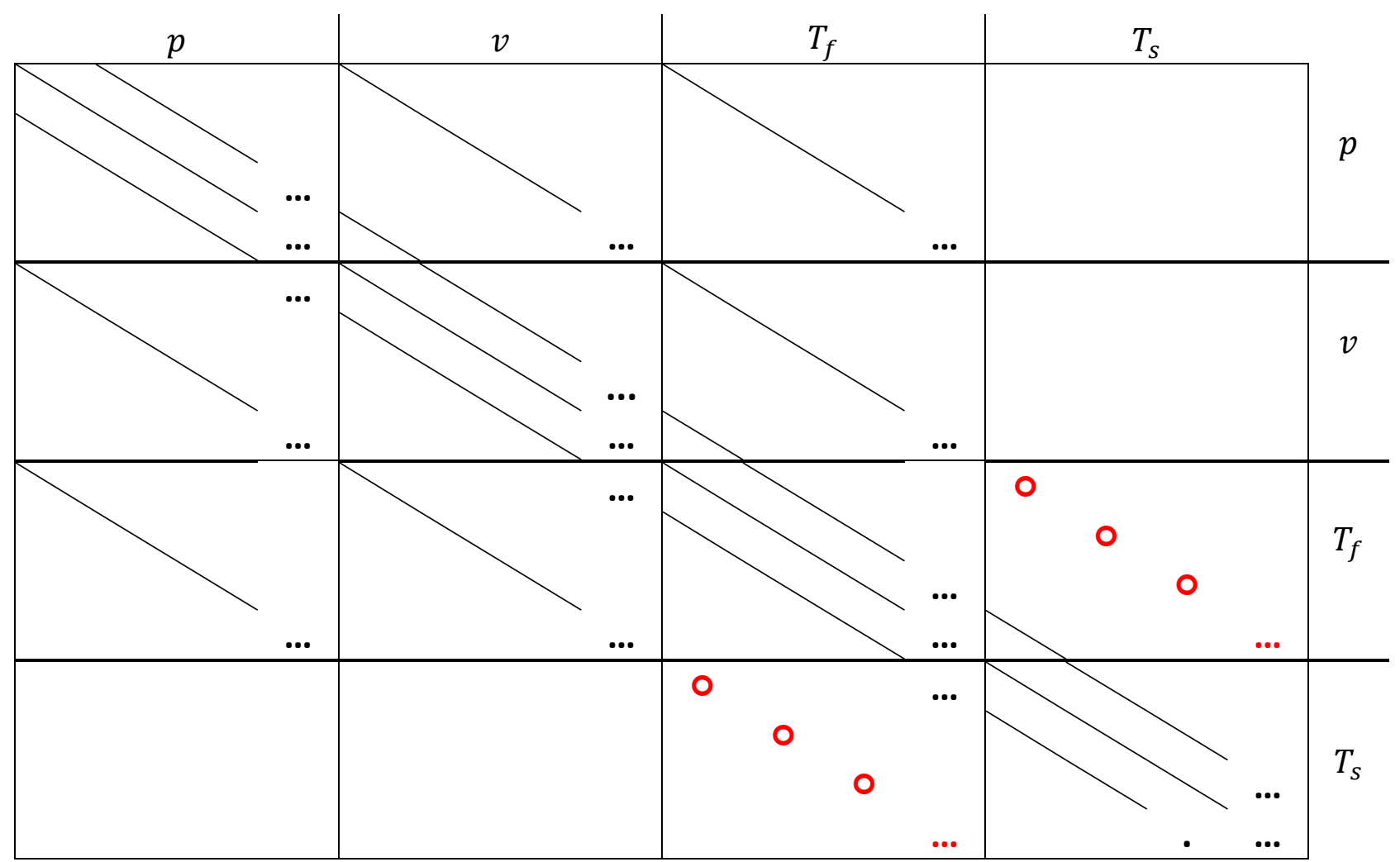

Figure 2: Preconditioning matrix for conjugate heat transfer problem (with lines, circles, and dots representing non-zero entries in the matrix)

\section{SAM MULTI-CHANNEL ROD BUNDLE MODEL}

To improve the heat transfer between the duct wall and coolant flow, a multi-channel rod bundle model is developed in SAM to account for the temperature differences between the center region and the edge region of the coolant channel in a fuel assembly. Similar approach has been proposed in ENERGY (Yang and Joo, 1999), SAS4A/SASSYS-1 (Fanning 2012), as well as the multi-region porous medium model reported by Yu et al. (2015). The whole fuel assembly can be divided into a number of regions, as shown in Figure 3. It is quite remarkable that the volumetric heat flux in region 1 is significantly less than that in other regions, based on the analytical calculation. Each inner region is modeled as an average corechannel (i.e., a 1-D coolant channel and an average fuel pin). The edge region can be modeled as one core-channel or six core-channels to account for the differences in heat transfer with each side of the duct wall. This zoning strategy is also inspired from the authors' previous experiences in the CFD simulations of the triangle-lattice pin bundles. As shown in Figure 4, large temperature gradient were observed in the 
coolant region near the duct wall, but the temperature distribution elsewhere is very uniform except the hot spots due to the wire-wrap effects.

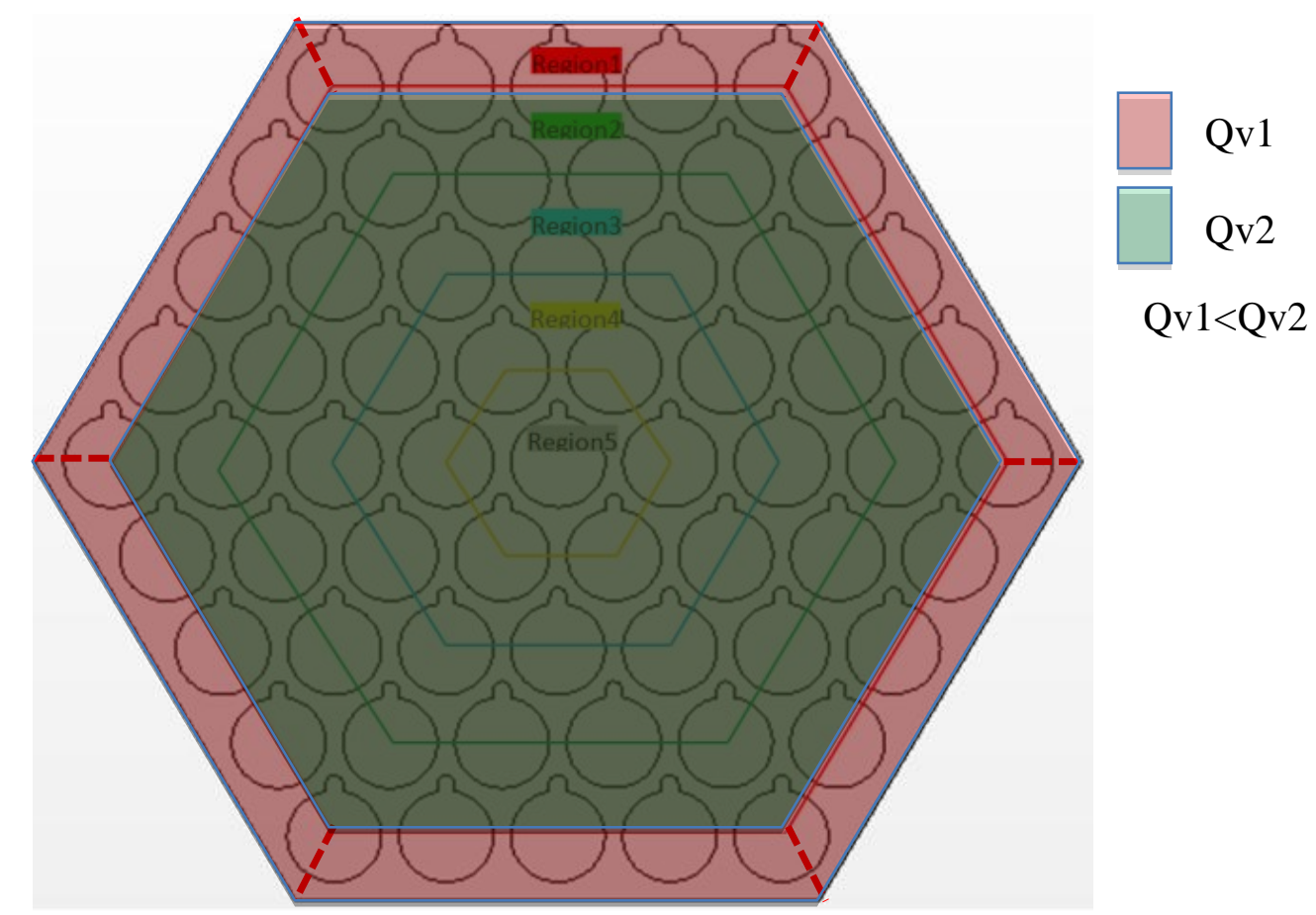

Figure 3: Sketch of the regions in the multi-channel model

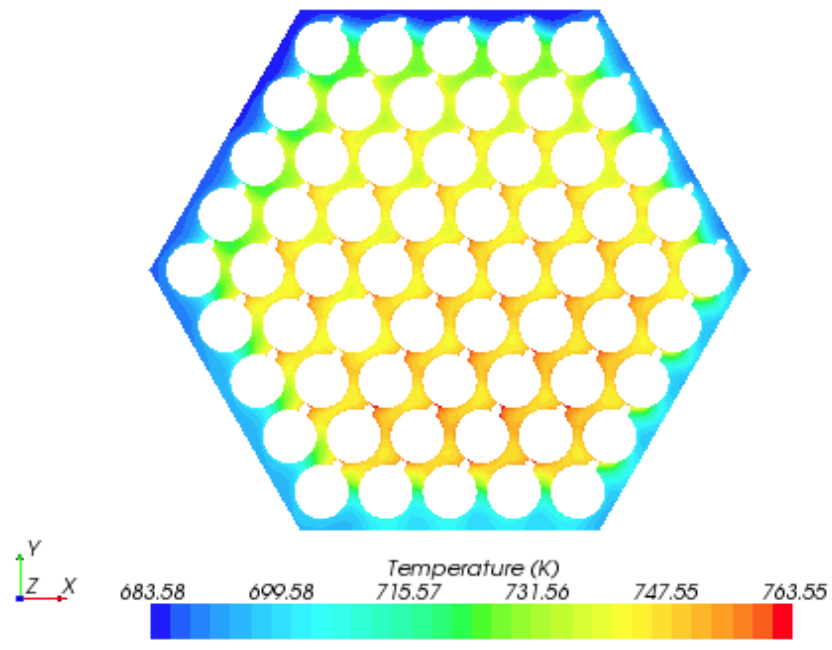

(a) 61-pin wire-wrapped bundle

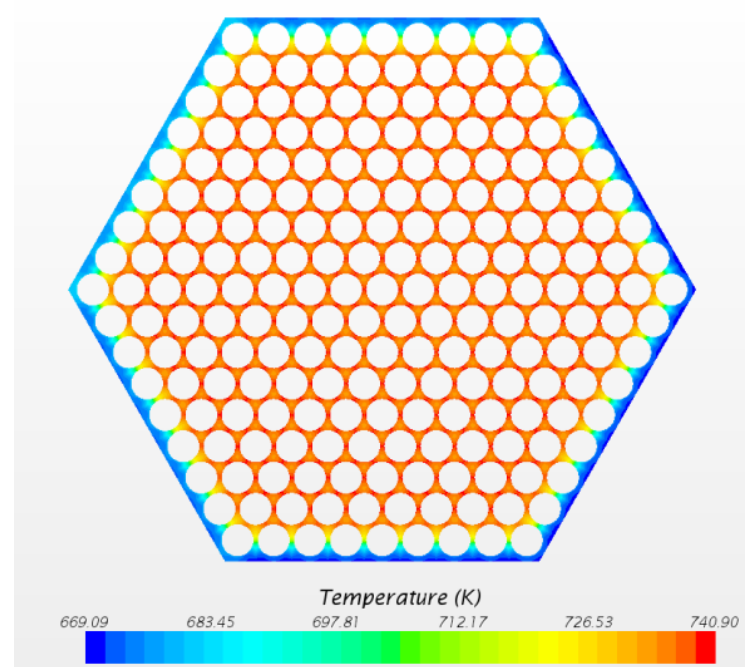

(b) 217-pin bare bundle

Figure 4: Typical coolant temperature distributions in rod bundles

In the SAM multi-channel model, the fluid regions are modeled as separate parallel channels with the same pressure drop. For simplicity, it is assumed that there are no mass and momentum exchange between channels. However, the energy exchange is allowed at all axial nodes, and the energy exchange rate is modeled as: 


$$
\frac{d \dot{Q}}{d z}=\beta(\rho v)_{a v g} S\left(h_{1}-h_{2}\right)
$$

in which, $\beta$ is the mixing parameter (accounting for both turbulent mixing and directional flow); ( $\rho v)_{\text {avg }}$ is the average mass flux between Region 1 and 2; $S$ the total gap width between Region 1 and 2; and $h_{1}$ and $h_{2}$ are the enthalpies of Region 1 and 2.

This term is modeled as an additional heat source term in the energy conservation equation,

$$
q_{\text {mix }}^{\prime \prime \prime}=\frac{1}{A_{c}} \frac{d \dot{Q}}{d z}=\beta(\rho v)_{a v g}\left(h_{1}-h_{2}\right) \frac{S}{A_{c}} \text {. }
$$

Because the SUPG stabilization scheme is used in SAM, the associated stabilization term need to be included in the residual calculation for each finite element:

$$
\Delta r_{\text {energy }}=\int_{\Omega} \psi * q_{\text {mix }}^{\prime \prime \prime} d \Omega=\sum_{i, q p}\left(q_{\text {mix }}^{\prime \prime \prime} \times\left(\phi+\tau_{\text {supg }} \cdot \nabla \phi\right)\right),
$$

In which, $i$ is the index of the test functions and $q p$ is the index of quadrature points for the element.

\section{DEMONSTRATION CALCULATION OF 7 FUEL ASSEMBLIES}

\subsection{Model Description}

A 7-assembly model has been developed to examine the pseudo 3-D full-core conjugate heat transfer modeling capability in SAM. The fuel assembly geometry is based on the Advanced Burner Test Reactor (ABTR) conceptual design (Chang et al., 2005), and the major parameters of the ABTR fuel assembly design are listed in Table 1.

Table 1: ABTR Fuel Assembly Parameters

\begin{tabular}{|l|c|}
\hline Assembly Parameters & \\
Pin number & 217 \\
Assembly pitch (m) & 0.14598 \\
Duct outside flat-to-flat distance (m) & 0.14198 \\
Duct inner flat-to-flat distance (m) & 0.13598 \\
Assembly duct thickness (m) & 0.003 \\
Inter-assembly gap width (m) & 0.004 \\
Assembly length (m) & 0.8 \\
\hline Pin Parameters & \\
Pin diameter (m) & 0.008 \\
Pin pitch-to-diameter ratio & 1.13 \\
Pin pitch (m) & 0.00904 \\
\hline
\end{tabular}

Seven identical fuel assemblies of 217 pins each are modeled in this study. The red dots in Figure 5a represent the fluid nodes; the 12 blue lines between two fluid nodes represent all the heat structures between two assemblies (including two duct wall widths and the inter-assembly sodium gap); and 6 other blue lines near the fluid nodes represent the duct wall sides with adiabatic boundary conditions on the outside surface. For simplicity, the inter-assembly flow in the gap is neglected in this work, and only heat conduction is considered. A CFD model is also developed for comparison, as seen in Figure 5b. For simplicity (in the CFD simulation), only bare-bundle simulations were performed in this code-to-code benchmark exercise, and constant thermophysical properties of the sodium and duct wall are used in this work. 
In the 7-assembly model, it is assumed that the center assembly (\#0) has higher power density with power peaking factor of 1.5, and that the lower-right assembly (\#6) has lower power density with power peaking factor of 0.5. All the other assemblies have the same power density with power peaking factor of 1 . Uniform power distributions (both radial and axial) are assumed within each assembly. Additionally, the same inlet flow rate is applied for all assemblies.

\section{Name rule:}

Number: assemble position (0 6)

Alphabet: wall position ( $a, b, c, d, e, f)$

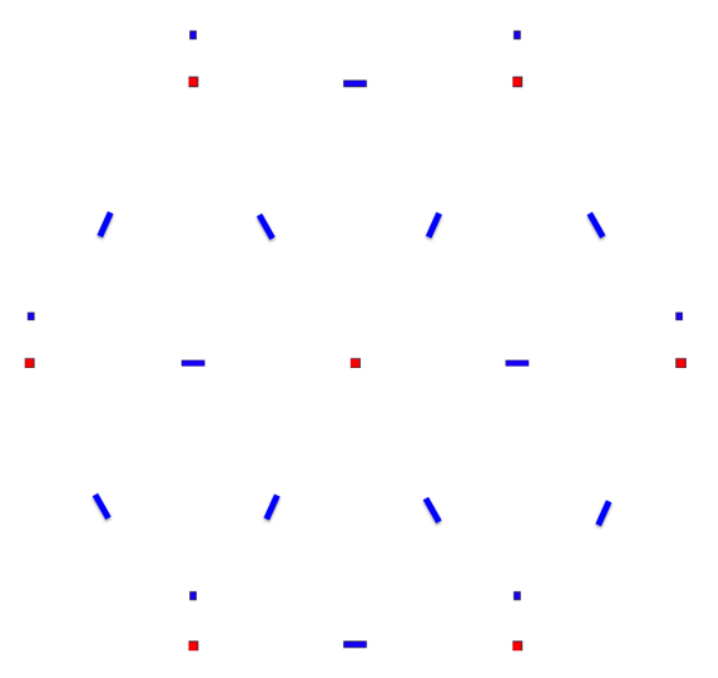

(a) Single-Channel System TH model

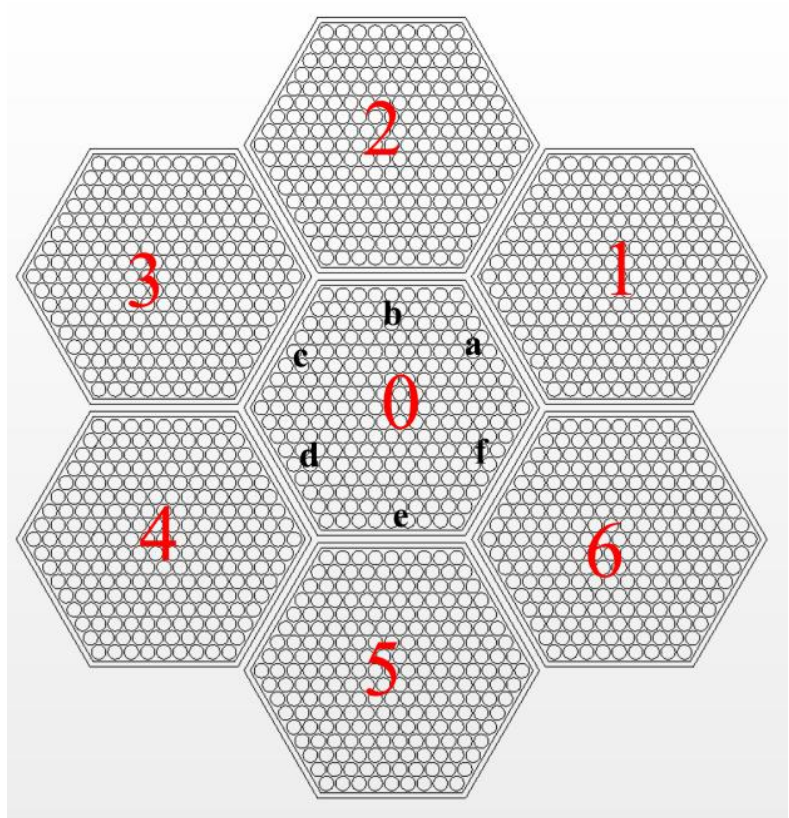

(b) CFD model

Figure 5: The 7-assembly computational model (top view) and notations

\subsection{CFD Simulation Results}

Realizable k- $\varepsilon$ turbulence model, the two-layer all-y+ wall formulation, and segregated flow solver with the SIMPLE predictor-corrector algorithm are used in the CFD simulation. The solution is well converged as the normalized residuals are below $10^{-4}$. Figure 6 presents the temperature distributions at the core outlet of the 7-assembly CFD simulation. It is seen that the hot and cold assemblies significantly affected the duct wall temperatures of the neighbor assemblies. However, the effect diminishes with increasing distance from neighbor assembly ducts to these two assemblies. 


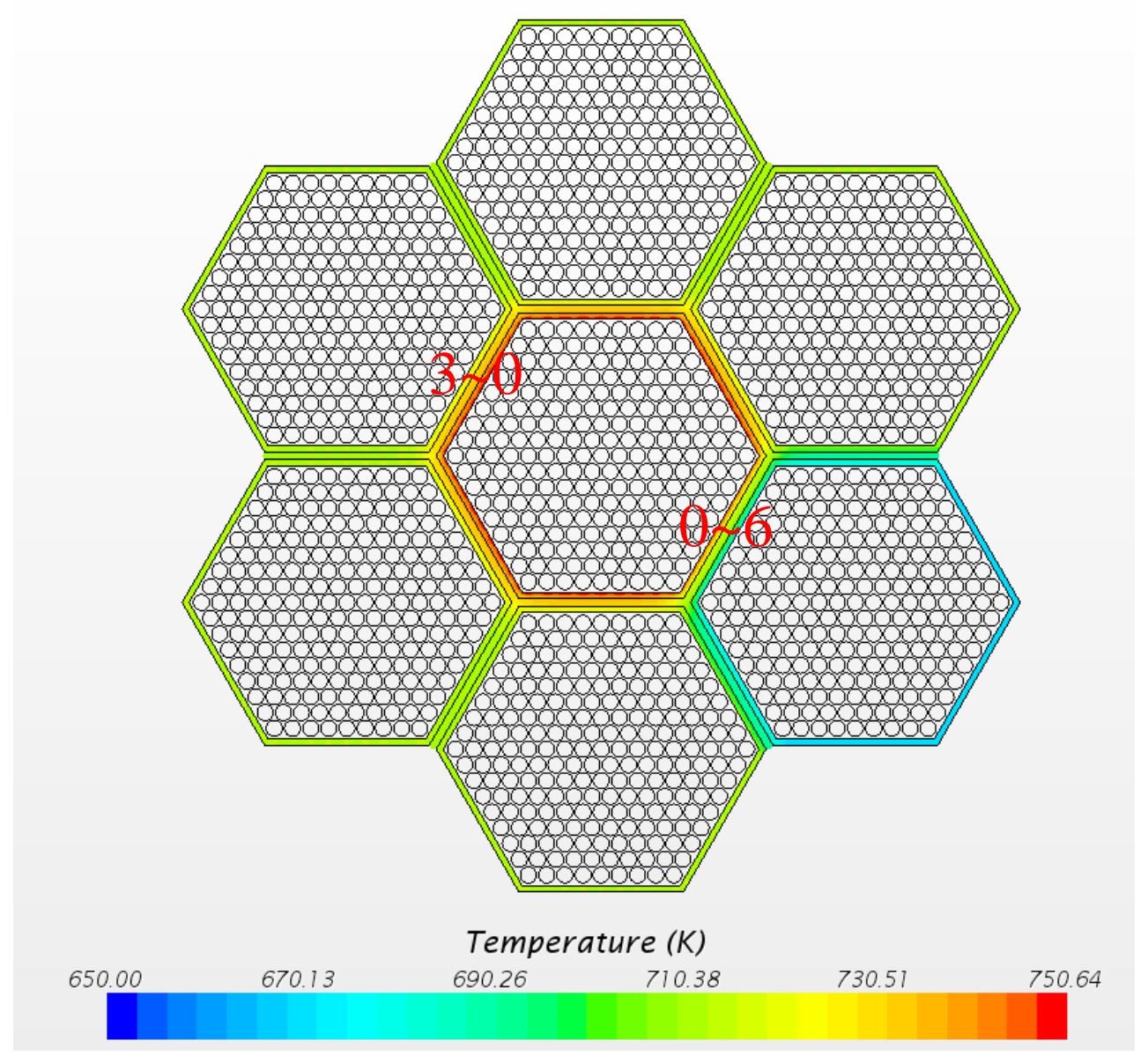

(a) Duct walls and assembly gap temperature distribution

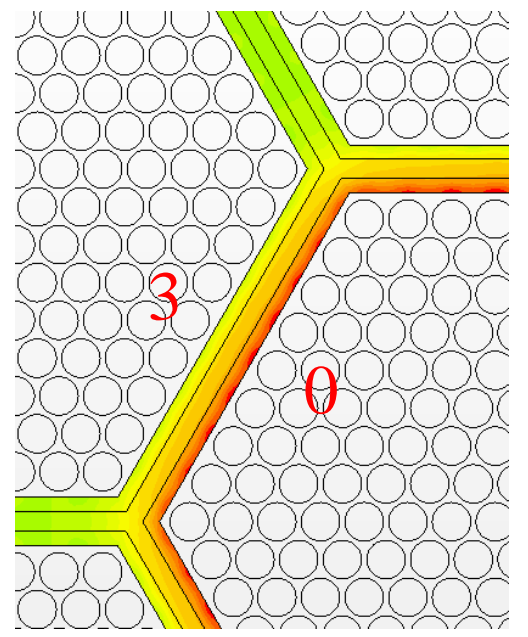

(b) Between assembly \#0 and \#3

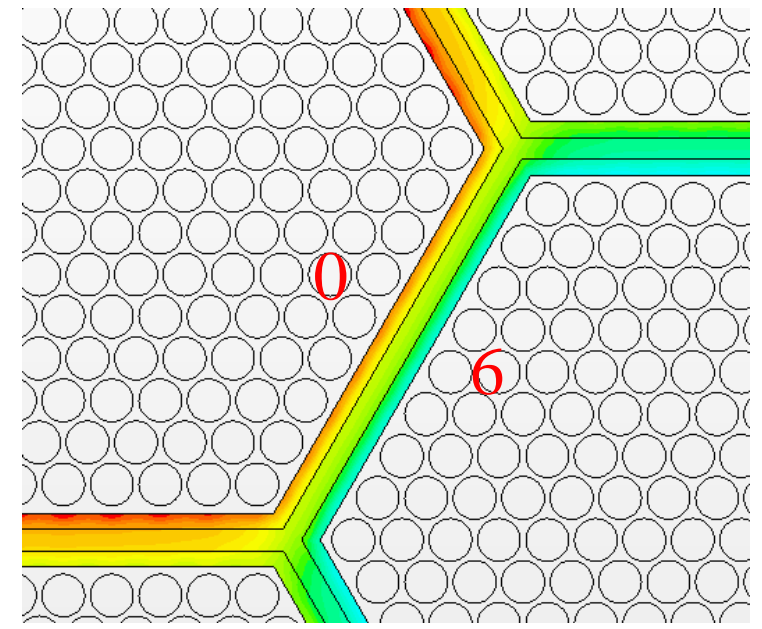

(c) Between assembly \#0 and \#6

Figure 6: Duct wall temperature distributions at the core outlet of 7-assembly CFD simulation 


\subsection{Single-Channel Model Results}

The single-channel approach was first applied in SAM to confirm its poor performance in predicting the duct wall temperature. The average axial temperature distributions from SAM and CFD simulations are compared in Figure 7. It is seen that the average coolant temperature predictions in the high power channel (Assembly 0) and low power channel (Assembly 6) agrees very well between the two approaches. However, large differences were observed for the duct wall temperatures. It is noted that inner temperature of Side $\mathrm{C}$ of Assembly 6 duct wall is lower than the average coolant temperature in CFD simulation, while it is higher than the coolant temperature in the SAM simulation.

To better understand the differences between the two approaches, the coolant temperature distributions at the core outlet of Assembly 0 and 6 from the CFD simulation are shown in Figure 8. It is seen that the edge region of the two assemblies are much colder than the inner regions, as expected. It is also confirmed that the temperature of duct wall $6 \mathrm{C}$ is higher than the coolant temperature near the wall in the CFD simulation; therefore, the coolant of Assembly 6 is receiving heat from the wall $6 \mathrm{C}$, although its average coolant temperature is higher than the wall. However, in the SAM simulation using SingleChannel approach, only one average coolant temperature is modeled per assembly. Therefore, to correctly model the heat transfer direction between the assemblies, the duct wall temperature predictions are significantly overestimated in the SAM simulation.

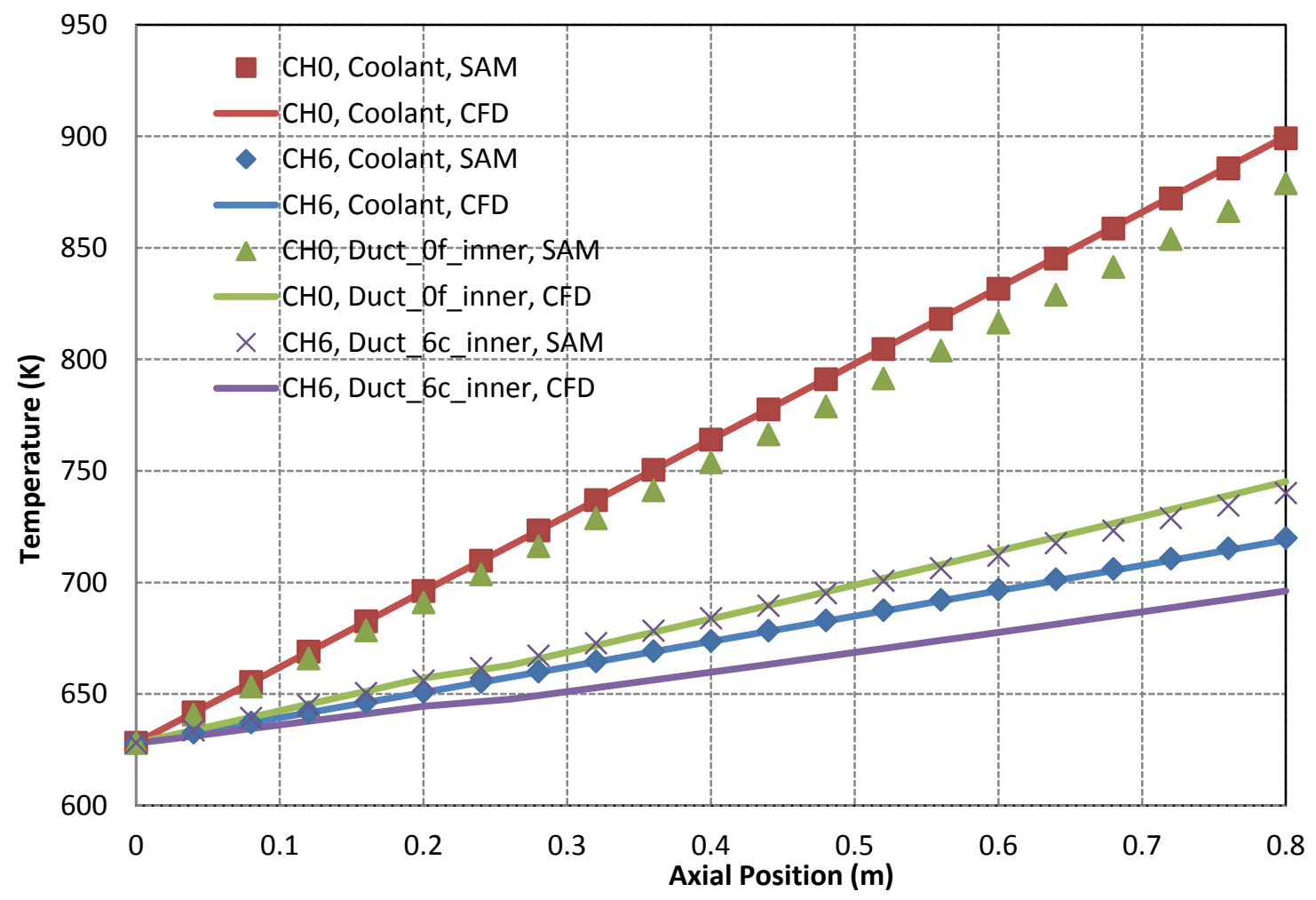

Figure 7: Comparison of average axial temperature distributions between SAM and CFD 


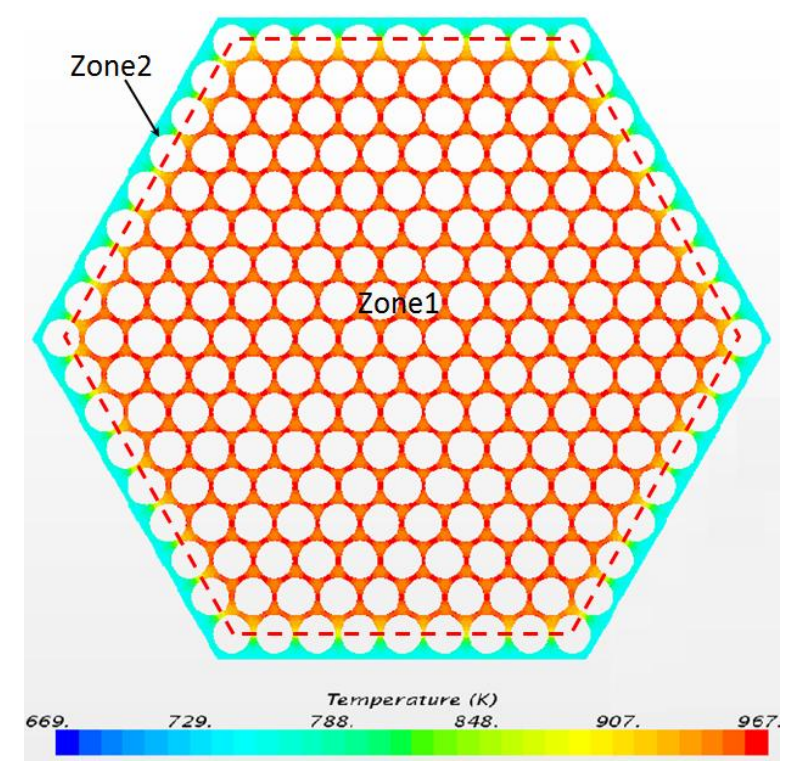

(a) Assembly 0

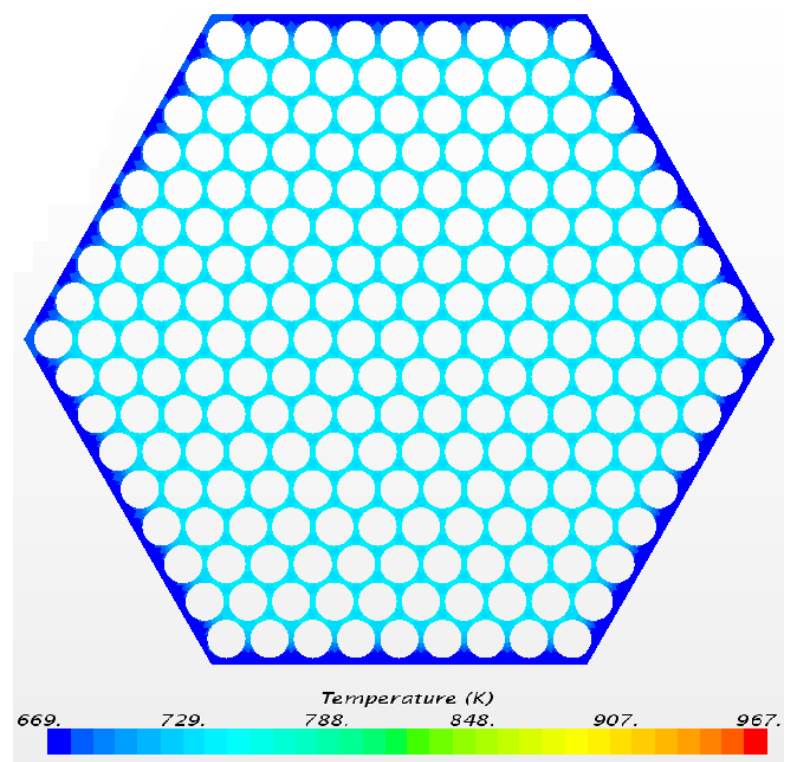

(b) Assembly 6

Figure 8: Coolant temperature distribution at core outlet of Assembly 0 and 6

\subsection{Results of Two-Region Two-Channel Model}

The two-region two-channel approach was then applied in SAM to examine its performance in predicting the duct wall temperature. Based on an energy balance calculation using the CFD simulation results, it is found that the energy exchange between the inner and edge zones is very small comparing to the heating power in each zone for the 7-assembly test problem. Therefore, $\beta=0$ was assumed in the SAM analysis of this demonstration problem.

The axial temperature distributions of the Assembly 0 and 6 from the SAM two-region model simulation are shown in Figure 9. Note that the temperature differences between the inner wall and the edge coolant are the same for Assembly 0 and 6, indicating the convective heat transfer is balanced at the two sides of the inter-assembly heat structures. It is seen that the inner wall temperature of Duct $6 \mathrm{C}$ is lower than the average coolant temperature of Assembly 6, but higher than the edge coolant temperature, as observed in the CFD simulation. In the two-region model, Zone 2 (edge region) has lower power density (for the total volume of pin and coolant regions), but higher mass flux (due to the larger hydraulic diameter and less friction coefficient). Therefore, its temperature would be much lower than that of Zone 1 (inner region). Since the duct wall would only interact with the edge zone, the wall temperature predictions would be much lower comparing to those in the simulation using the single-channel model, as observed from Figure 7 and Figure 9.

The radial temperature distributions of the six sides of Assembly 0 duct wall at the core outlet are shown in Figure 10. It is seen the temperature of Duct $0 \mathrm{~F}$ is significantly lower than the other sides, as it faced the lower power Assembly 6. The temperature differences among the other 5 sidewalls are negligible. This is because the inter-assembly heat transfer is very small compared to the heating power from the fuel rod, and the 5 average-power assemblies have almost the same coolant temperature predictions despite their relative positions to the high- or low-power assembly. For the center high-power assembly, the total heat removal between coolant and the six sides of the duct is $\sim 22.5 \mathrm{~kW}$, which is only $\sim 0.3 \%$ of the heating power (7.58 MW). 
The axial temperature distributions from the SAM two-region two-channel model are compared with the average temperatures from the CFD simulation results, as shown in Figure 11. The radial wall temperature distributions of the heat structure between Assembly 0 and Assembly 6 at the core outlet are shown in Figure 12. Linear temperature distributions are observed in the Duct 0F, the inter-assembly sodium gap, and duct 6C in both SAM and STAR-CCM+ simulations. Very good agreements have been achieved between the two approaches. It should be noted that the SAM code is very efficient as it only takes a few seconds on a single processor for this demonstration simulation, while the CFD simulation takes $\sim 18$ hours on 128 processors.

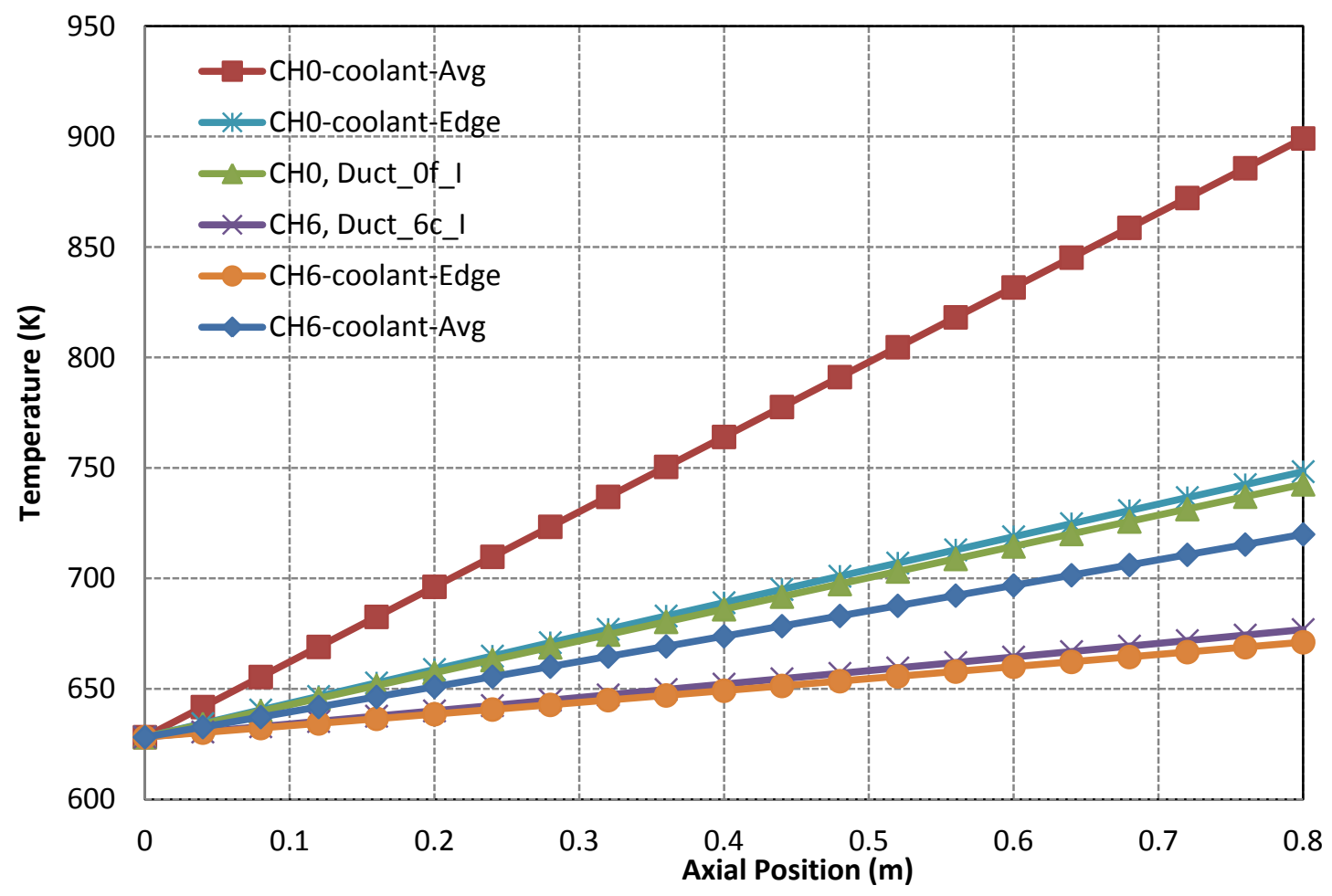

Figure 9: Axial temperature distributions of the Assembly 0 and 6, SAM two-region model 


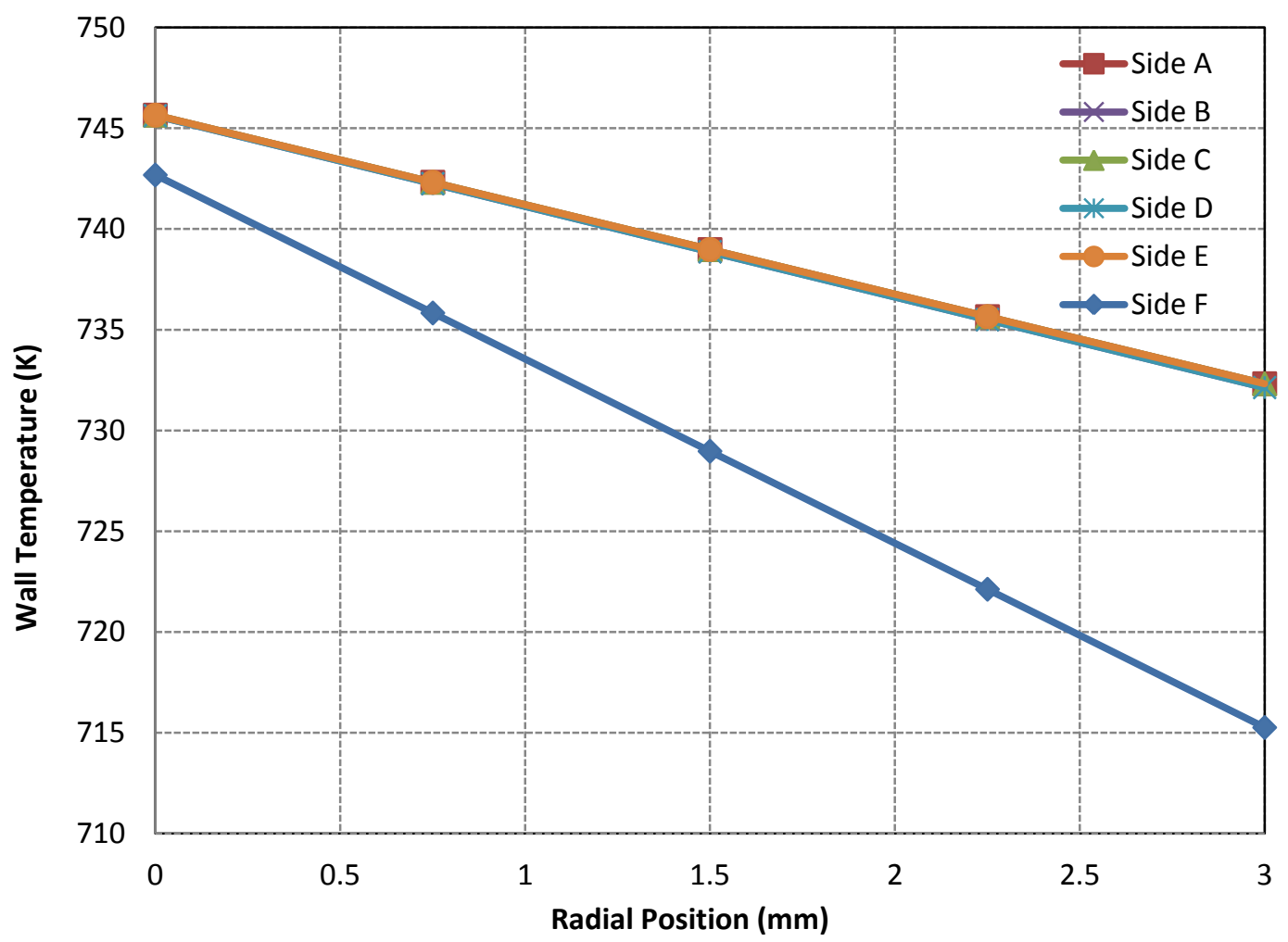

Figure 10: Radial temperature distributions of the six sides of Assembly 0 duct wall, at the core outlet

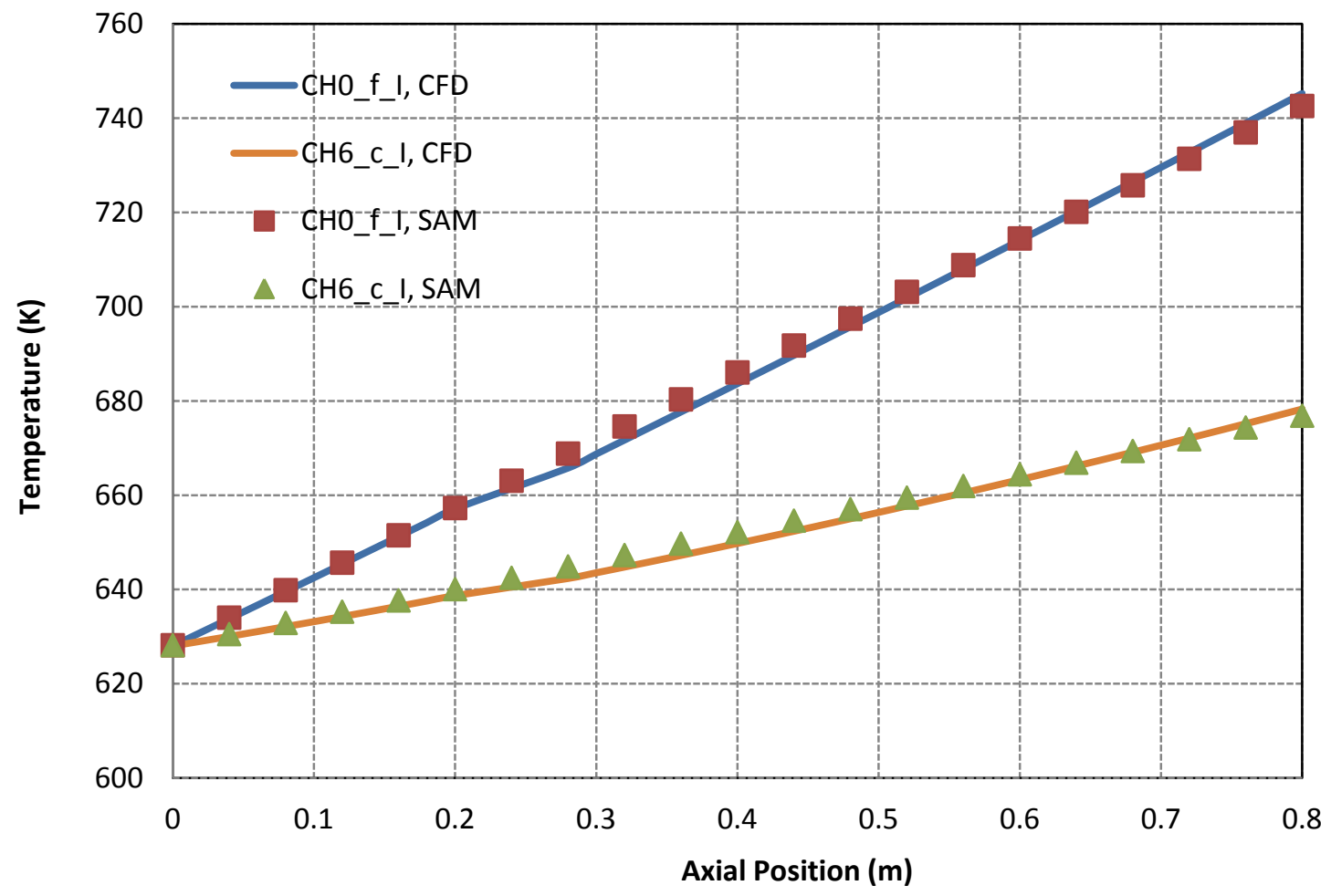

Figure 11: Comparison of average axial wall temperature distributions between SAM 2-region model and CFD 


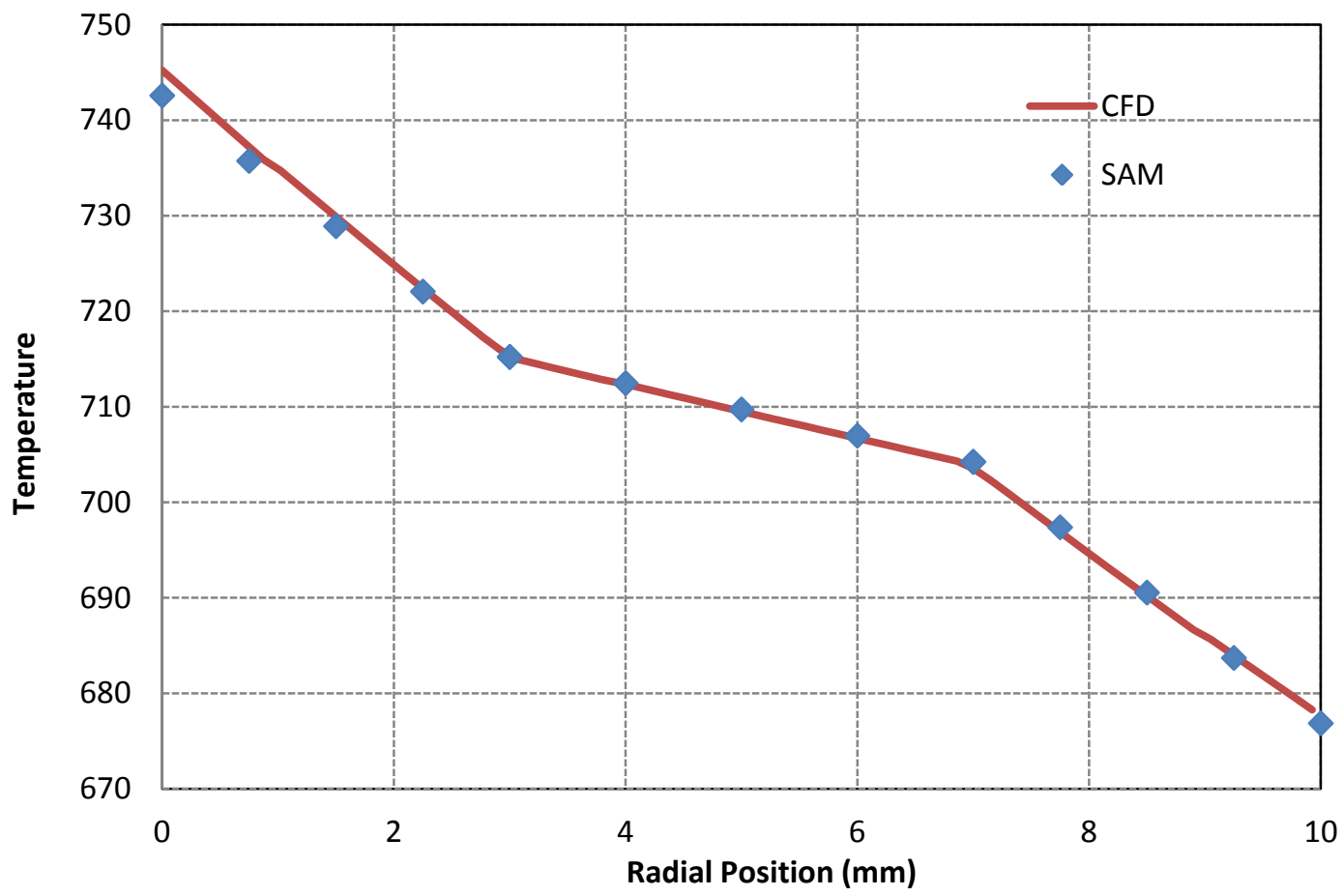

Figure 12: Comparison of radial wall temperature distributions between SAM 2-region model and CFD, heat structure between channel 0 and channel 6 at the core outlet

\subsection{Results of a Two-Region Seven-Channel Model}

As discussed above, although a single coolant temperature is used for the edge region with the two-region two-channel model, the average duct wall temperatures of each side of the fuel assembly can be accurately predicted during normal operating conditions. This is primarily due to the insignificant radial heat transfer between fuel assemblies, comparing to the heat generation in the fuel pins. However, this may not be the case during reactor transient or accidents when the reactor power is low and the temperature gradients among fuel assemblies are high. To examine the effects of the spatial discretization in the coolant region, the above seven-assembly test problem is modeled with a two-region seven-channel model, i.e., the edge coolant region is modeled as six channels.

The Two-Region Seven-Channel model was firstly applied to the same conditions of the 7-assembly test problem. The temperature distributions of all Assembly 0 and 6 coolant channels and the inter-assembly structure between the two assemblies are shown in Figure 13. Seven 1-D parallel channels were used to model the subassembly flow in both Assembly 0 and 6. It is seen that the central coolant channels are much hotter than the edge channels in both assemblies.

The axial temperature distributions of the six edge channels of Assembly 0 and 6 are shown in Figure 14 and Figure 15, respectively. Some differences are observed among the six edge regions. For Assembly 0 (high-power), the maximum differences among all sides are $2.6{ }^{\circ} \mathrm{C}$ in coolant temperature predictions, and $4.8{ }^{\circ} \mathrm{C}$ in inner duct wall temperature predictions. For Assembly 6 (low-power), the maximum differences among all six edge regions are $5.2{ }^{\circ} \mathrm{C}$ in coolant temperature predictions, and $9.6{ }^{\circ} \mathrm{C}$ in inner duct wall temperature predictions. Figure 16 shows the comparison of average axial wall temperature distributions (Duct OF and 6C) from SAM 2-region 7-channel (2R7C) model, SAM 2-region 2-channel (2R2C) model, and CFD results. Very small differences were observed among the three approaches. A deeper look at the simulation results found that the edge coolant temperature predictions of Assembly 6 
(low-power) in the two-region two-channel model are similar to the hottest side channel (Side C) in the two-region seven-channel model; while the edge coolant temperature predictions of Assembly 0 (highpower) in the two-region two-channel model are similar to the coldest side channel (Side F) in the tworegion seven-channel model.

It can be concluded that the SAM multi-channel model can accurately and efficiently predict the duct wall temperatures in a 3-D core lattice layout. The two-region two-channel model may accurately predict the inter-assembly heat transfer in the regions with highest temperature gradients; however, the two-region seven-channel model is preferred for accurate temperature predictions of the whole core. Some key information on the performance of various computational models is shown in Table 2. With the SAM multi-channel models (2R2C and 2R7C), the Degrees of Freedom (DOF) of the problem are significantly reduced, and the computational cost are reduced by 5-6 orders of magnitude from CFD simulations.

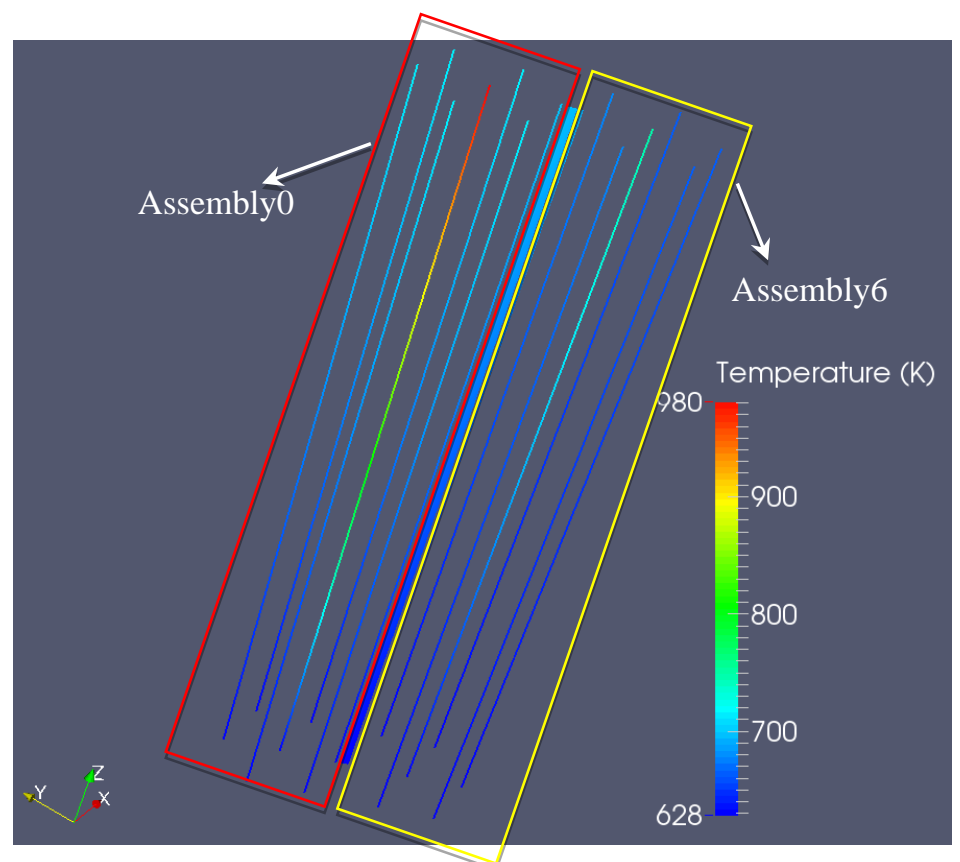

Figure 13: SAM temperature distributions of Assembly 0 and 6 coolant channels, and the inter-assembly structure

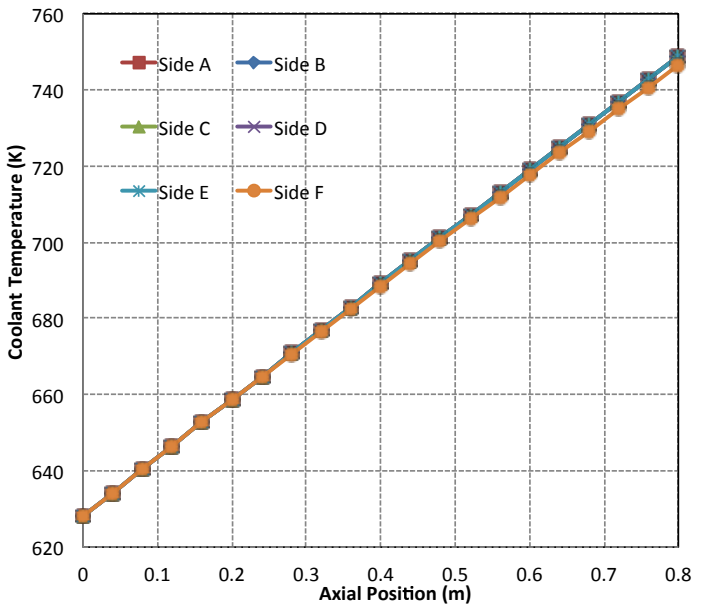

(a) Coolant temperature

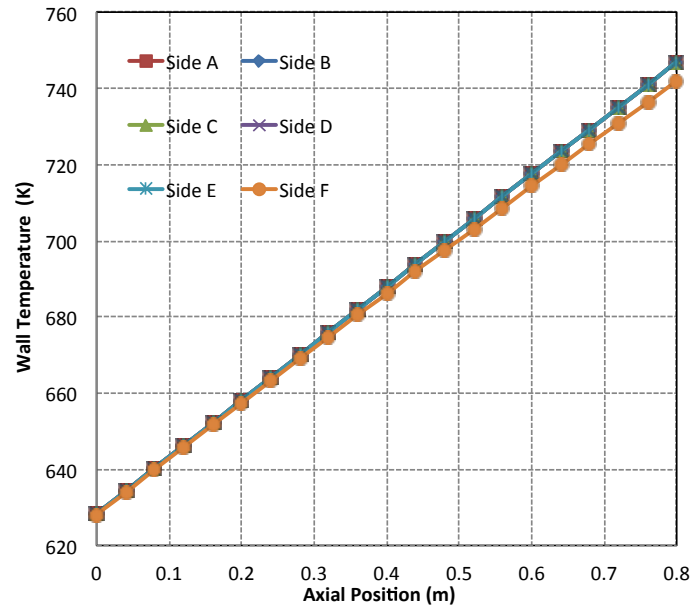

(b) Inner Wall Temperature

Figure 14: Axial temperature distributions of the center assembly edge region, 2-region 7-channel model 


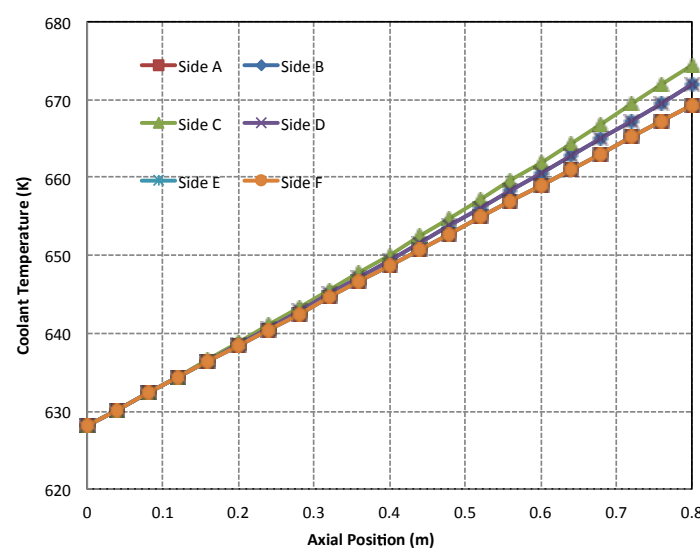

(a) Coolant temperature

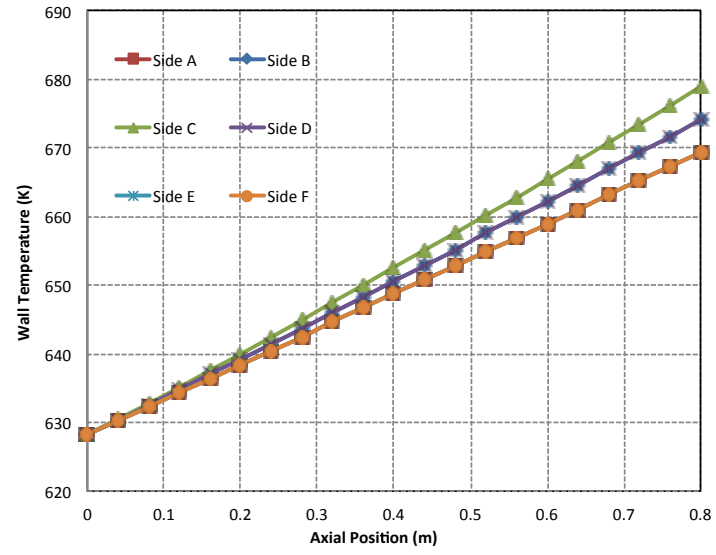

(b) Inner Wall Temperature

Figure 15: Axial temperature distributions of Assembly 6 edge region, 2-region 7-channel model

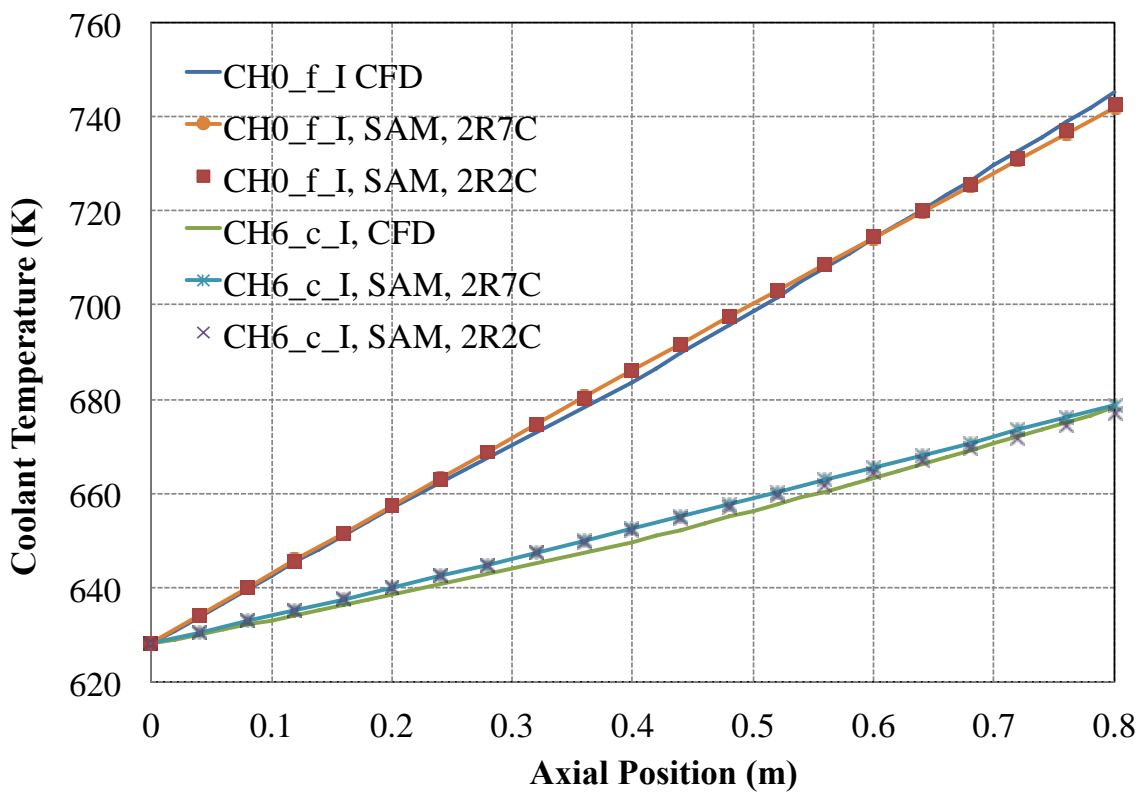

Figure 16: Comparison of average axial wall temperature distributions between SAM 2-region 7-channel model, 2-region 2-channel model, and the CFD model

Table 2: The performance of various computational models

\begin{tabular}{|l|c|c|c|c|}
\hline \multirow{2}{*}{ Computation Models } & $\begin{array}{c}\text { Degrees of Freedom } \\
\text { (DOF) }\end{array}$ & Computational cost & \multicolumn{2}{|c|}{$\begin{array}{c}\text { Average wall temperature } \\
\text { at core outlet (K) }\end{array}$} \\
\cline { 3 - 5 } & 71 million & $\begin{array}{c}\sim 2300 \text { core-hours or } \\
8.3 \text { million seconds }\end{array}$ & 745 & 679 \\
\hline CFD & 4227 & 2 seconds & 879 & 740 \\
\hline SAM - SingleChannel & 6285 & 8 seconds & 743 & 677 \\
\hline SAM - 2R2C & 16575 & 44 seconds & 742 & 679 \\
\hline SAM - 2R7C & &
\end{tabular}


To further examine the effects of the spatial discretization in the coolant region, the test problem is modified to low-power and low-flow conditions when the radial heat transfer between assemblies is more important to the coolant temperature predictions near the wall. Both power and flow rates are at $2 \%$ of the normal operating conditions for each assembly.

The axial temperature distributions of the six edge channels of Assembly 0 and 6 are shown in Figure 17 and Figure 18, respectively. Significant differences are observed among the six edge regions. For Assembly 0 (high-power), the maximum differences among all sides are $\sim 15{ }^{\circ} \mathrm{C}$ in both coolant and inner duct wall temperature predictions. For Assembly 6 (low-power), the maximum differences among all six edge regions are $\sim 30^{\circ} \mathrm{C}$ in both coolant and inner duct wall temperature predictions.

Figure 19 shows the radial temperature distributions of the heat structures between Assembly 0 and 6 and between Assembly 5 and 6 at the core outlet. Very different predictions were obtained from the 2-region 7-channel model and the 2-region 2-channel model. The differences in temperature predictions of the structure between Assembly 5 and 6 are $\sim 15{ }^{\circ} \mathrm{C}$ between the two modeling approaches. It clearly demonstrates the importance to model each side of the edge coolant region separately for accurate interassembly heat transfer modeling and structure temperature predictions.

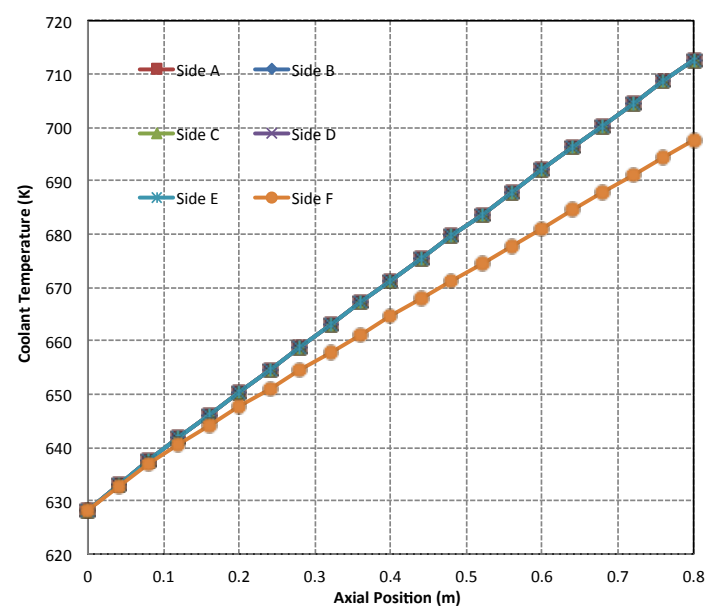

(a) Coolant temperature

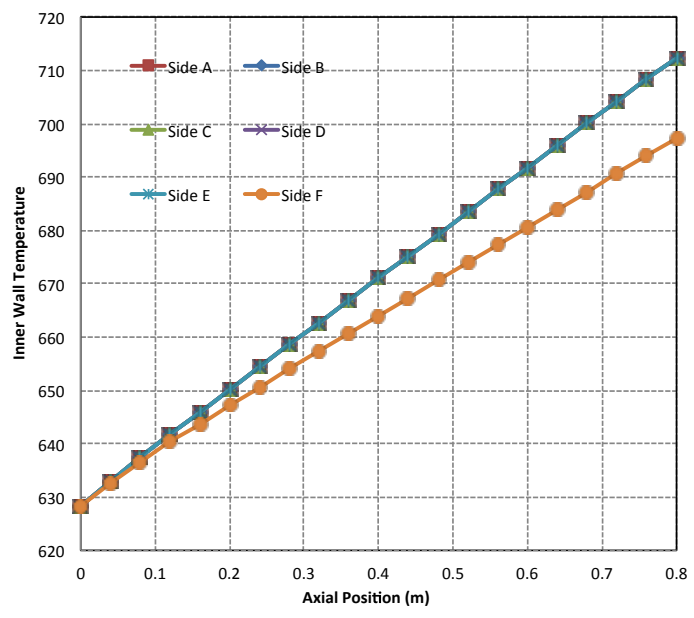

(b) Inner Wall Temperature

Figure 17: Axial temperature distributions of the center assembly edge region, low-power low-flow conditions

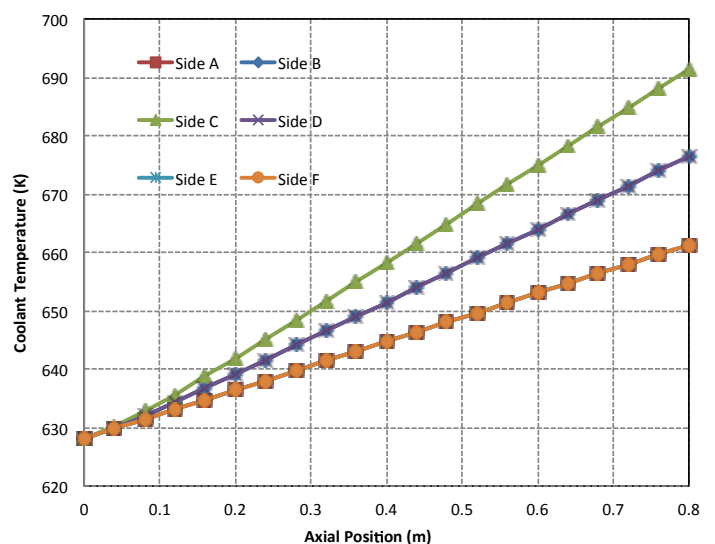

(a) Coolant temperature

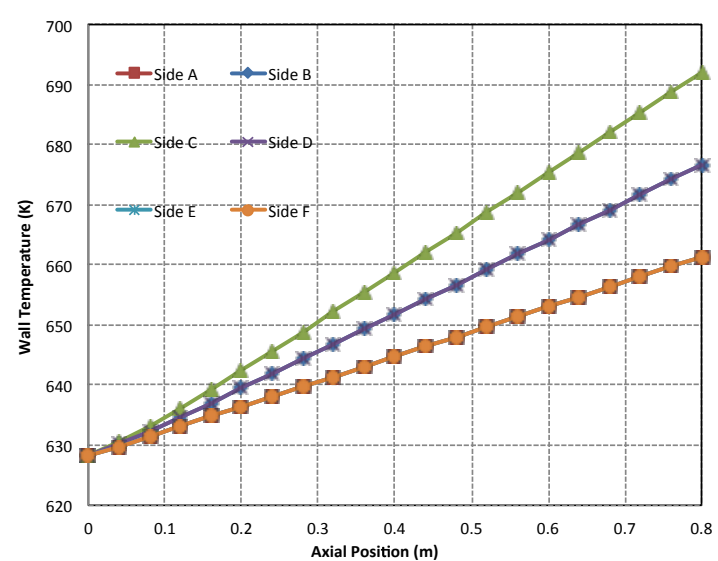

(b) Inner Wall Temperature

Figure 18: Axial temperature distributions of Assembly 6 edge region, low-power low-flow conditions 


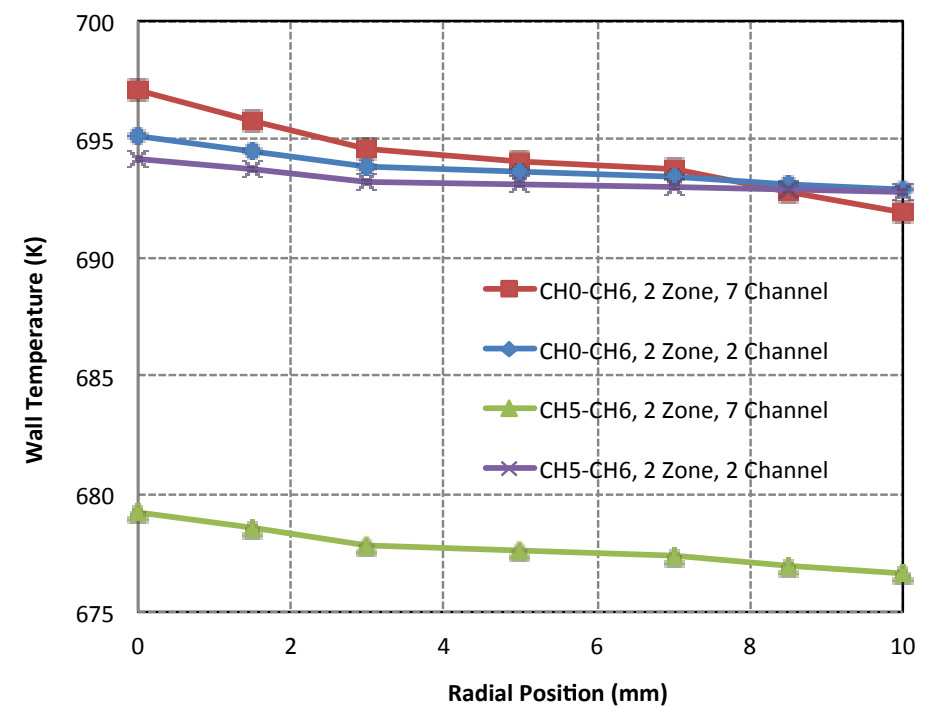

Figure 19: Comparison of radial wall temperature distributions between SAM 2-region 2-channel model and 2-region 7-channel model at the core outlet

\section{CONCLUSIONS}

A pseudo 3-D full-core conjugate heat transfer modeling capability has been developed in the advanced system analysis tool SAM for efficient and accurate temperature predictions of SFR structures. A multichannel rod bundle model is developed in SAM to account for the temperature differences between the center region and the edge region of the coolant channel in a fuel assembly. The edge region can be modeled as six core channels to account for the differences in heat transfer with each side of the duct wall. The hexagon lattice core is modeled with 1-D parallel channels representing the subassembly flow, and 2-D duct walls and inter-assembly gaps. The six sides of the hexagon duct wall are modeled separately to account for different temperatures and heat transfer between inner assembly flow and each side of the duct wall. A core lattice model is developed to facilitate the generation of all the core channels and inter-assembly gaps. The Jacobian Free Newton Krylov (JFNK) solution method is applied to solve the fluid and solid field simultaneously to avoid the operator-splitting errors, which is especially valuable for conjugate heat transfer modeling.

The 3-D full-core conjugate heat transfer modeling capability in SAM has been demonstrated by a verification test problem with 7 fuel assemblies in a hexagon lattice layout. The simulation results are compared with RANS-based CFD simulations. It was found that the single-channel model (one coolant temperature per axial position) would significantly overestimate the duct wall temperatures. Instead, a multi-channel rod-bundle model is required to accurately model the duct wall temperature and interassembly heat transfer. Using the two-region two-channel model, SAM predictions can agree well with the results from the CFD simulation in the regions with high temperature gradients and under high-power high-flow conditions. However, it is needed to model each side of the edge coolant region separately (such as in the two-region seven-channel model) for accurate inter-assembly heat transfer modeling and structure temperature predictions for the whole core under wide range of operating conditions. The computational cost in SAM simulations is very small, reduced by 5-6 orders of magnitude from CFD simulations. It can be concluded that the SAM can efficiently and accurately model the inter-assembly heat transfer and the duct wall temperatures in a 3-D core lattice layout. 


\section{ACKNOWLEDGMENTS}

This work is supported by U.S. DOE Office of Nuclear Energy's Nuclear Energy Advanced Modeling and Simulation (NEAMS) program. The submitted manuscript has been created by UChicago Argonne, LLC, Operator of Argonne National Laboratory ("Argonne”). Argonne, a U.S. Department of Energy Office of Science laboratory, is operated under Contract No. DE-AC02-06CH11357.

\section{REFERENCES}

1. Y. I. Chang, P. J. Finck, C. Grandy, et al., "Advanced Burner Test Reactor Preconceptual Design Report," ANL-ABR-1 (ANL-AFCI-173), Argonne National Laboratory, September 2006.

2. R. Hu and T. H. Fanning, "A Momentum Source Model for Wire-Wrapped Rod Bundles - Concept, Validation, and Application”, Nuclear Engineering and Design, Vol. 262, 371-389, (2013).

3. F. Roelofs, V.R. Gopala, L. Chandra, et al. "Simulating fuel assemblies with low resolution CFD approaches." Nuclear Engineering and Design 250, 548-559, (2012).

4. M. Viellieber and A. Class, "Investigating Reactor Components with The Coarse-GridMethodology," Proceedings of NURETH-16, Chicago IL, USA, August 30-September 3, 2015.

5. W.S. Yang, A.M. Yacout, "Assessment of the SE2-ANL Code Using EBR-II Temperature Measurements," Proceedings or NURETH-7, Saratoga Springs, NY, September 10-15, 1995.

6. W.S. Kim, Y.G. Kim, Y.J. Kim, “A subchannel analysis code MATRA-LMR for wire wrapped LMR subassembly," Annals of Nuclear Energy, 29, pp. 303-321, (2002).

7. D. Tenchine, V. Barthel, U. Bieder, et al., "Status of TRIO_U code for sodium cooled fast reactors," Nuclear Engineering and Design, Vol. 242, 307-315, (2012).

8. Y. Yu, E. Merzari, A. Obabko, J. Thomas, "A porous medium model for predicting the duct wall temperature of sodium fast reactor fuel assembly," Nuclear Engineering and Design, Vol. 295, 48-58, (2015).

9. D. Tenchine, "Some thermal hydraulic challenges in sodium cooled fast reactors," Nuclear Engineering and Design, 240, 1195-1217, (2010).

10. R. Hu, T.H. Fanning, T. Sumner, Y. Yu, "Status Report on NEAMS System Analysis Module Development," ANL/NE-15/41, Argonne National Laboratory, December 2015.

11. R. Hu, "An Advanced One-Dimensional Finite Element Model for Incompressible Thermally Expandable Flow", Nuclear Technology, Vol. 190, No. 3, 313-322, (2015).

12. CD-adapco, "STAR-CCM+ v 9.04.009 User Manual,” New York, 2014.

13. D. Gaston, C. Newman, G. Hansen, and D. Lebrun-Grandi'e, "MOOSE: A parallel computational framework for coupled systems of nonlinear equations," Nuclear Engineering and Design, vol. 239, pp. 1768-1778, (2009).

14. B. S. Kirk, J. W. Peterson, R. H. Stogner, and G. F. Carey, "libMesh: A C++ Library for Parallel Adaptive Mesh Refinement/Coarsening Simulations," Engineering with Computers, 22(3-4): 237254, (2006).

15. S. Balay, J. Brown, et al, PETSc Web page, http://www.mcs.anl.gov/petsc, 2016.

16. R. Hu and T. Sumner, "Benchmark Simulations of the Thermal-Hydraulic Responses during EBR-II Inherent Safety Tests using SAM", Proceedings of ICAPP'16, San Francisco CA, April 17-20, 2016.

17. D. A. Knoll and D. E. Keyes, "Jacobian-free Newton-Krylov Methods: a Survey of Approaches and Applications," Journal of Computational Physics, Vol. 193, pp.357-397 (2004). 
18. L. Zou, J. Peterson, H. Zhao, et al., "Solving Implicit Multi-Mesh Flow and Conjugate Heat Transfer Problems with RELAP-7," Proceedings of M\&C 2013, Sun Valley, Idaho, May 5-9, 2013.

19. W.S. Yang and H.G. Joo, "LMR core temperature calculation based on implicit formulation of the ENERGY model and a Krylov subspace method," Annals of Nuclear Energy, Vol. 26, pp. 629-640 (1999).

20. T. H. Fanning, ed., "The SAS4A/SASSYS-1 Safety Analysis Code System," Argonne National Laboratory, ANL/NE-12/4, January 2012. 